\title{
Designing Dying Well
}

Toward a new architectural approach of in-patient palliative care environments for the terminally ill

Annie Bellamy $\mid \mathrm{PhD}$ candidate | Welsh School of Architecture bellamyas@cardiff.ac.uk

Marie Curie Annual Conference 2018 From radical to real: Implementing new models of palliative care 


\section{Design background}

$\mathrm{Ba}$ (Hons)

Central Saint Martins

M:Arch

Welsh School of Architecture

\section{Practice background}

5th Studio, Cambridge

Emmett Russell Architects, Bristol

Allies + Morrison, Cambridge 
"I should say: the house shelters day-dreaming, the house protects the dreamer, the house allows one to dream in peace."

Gaston Bachelard The Poetics of Space 


\section{the hospice in history}

Patients of the Hotel-Dieu in their Beds, folio 77, 1482, Livre de vie active, by Jehan Henry, shows nurses attending the dying in a Paris hospital. Patients are two to a bed, except for a dying woman

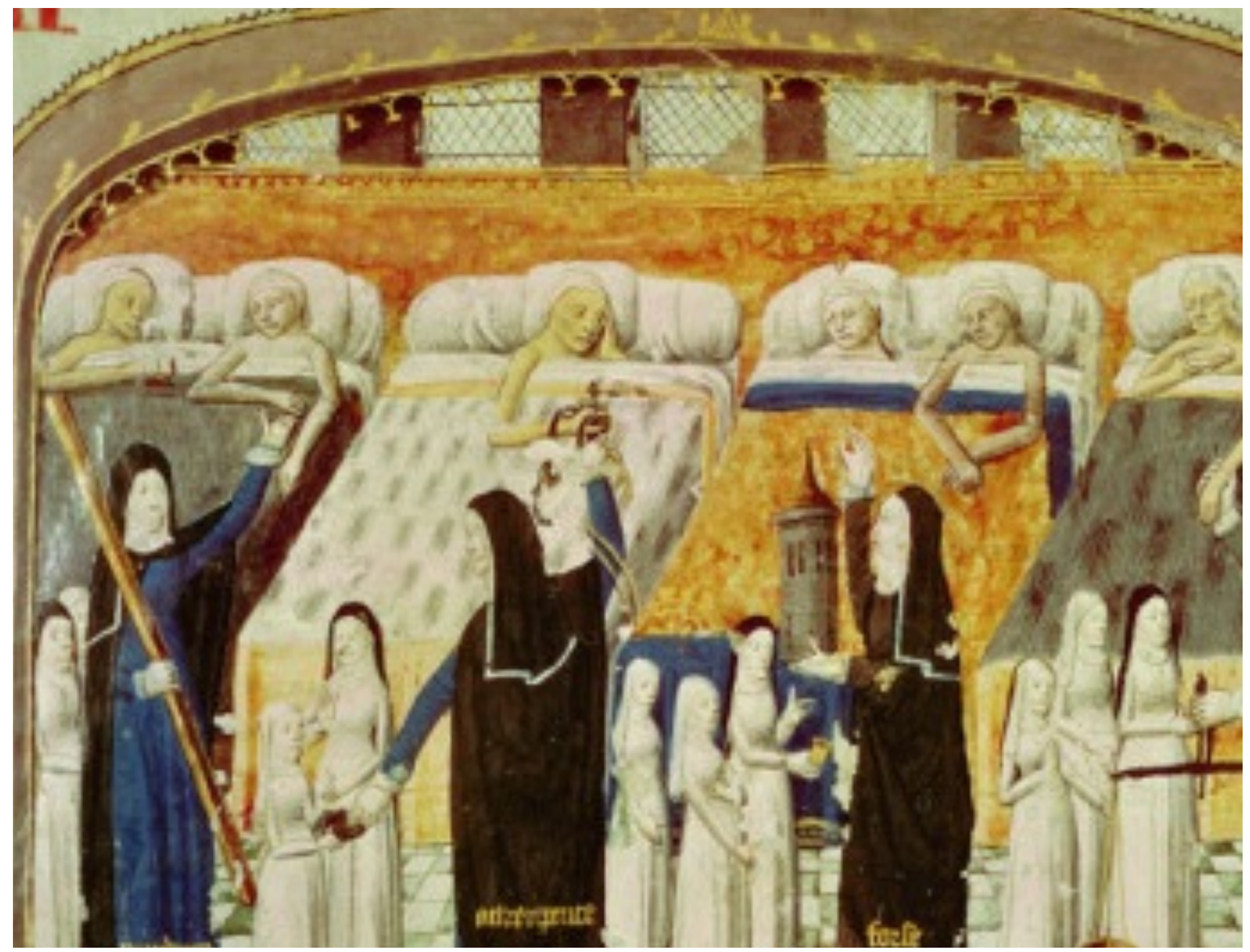




\section{the hospice now}
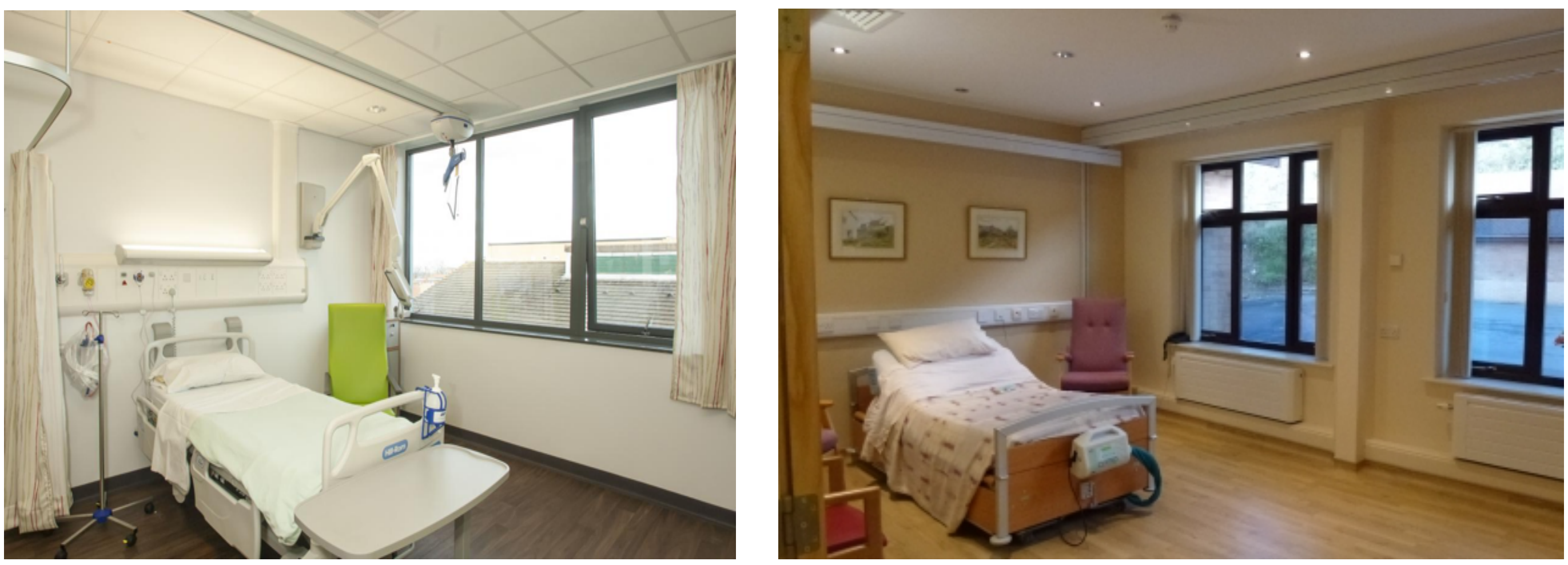

[https://stdavidshospice.org.uk/about-us/what-we-do/] [https://www.qegateshead.nhs.uk/node/1306] 


\title{
homely? home-like? home-liness?
}

\author{
home?
}


"architectural determinism works under some condition, but only to a degree, and it depends on the culture of the inhabitants and their other conditions of stress and well-being"

Charles Jencks, “Can Architecture Affect Your Health?” pg 14 


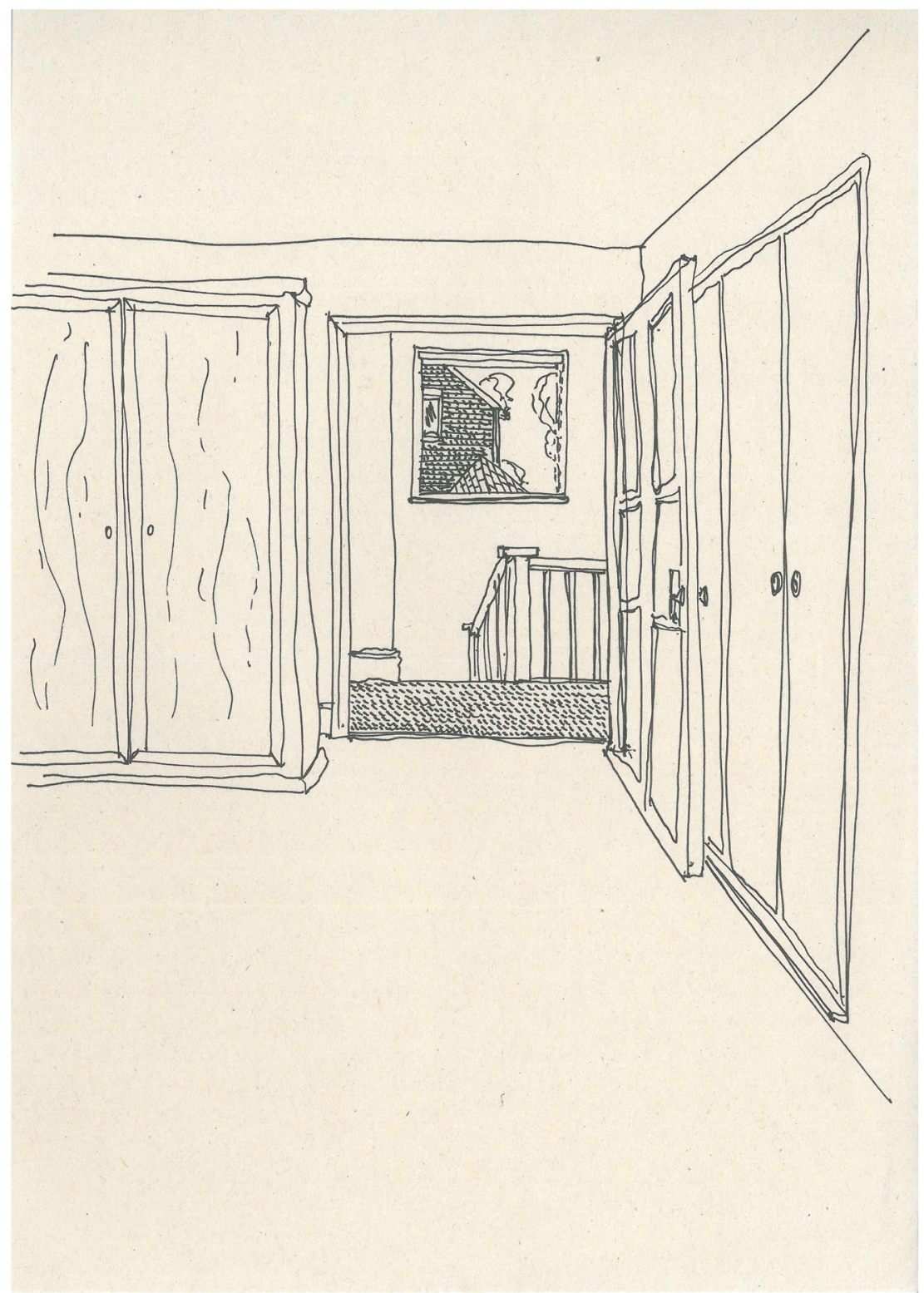




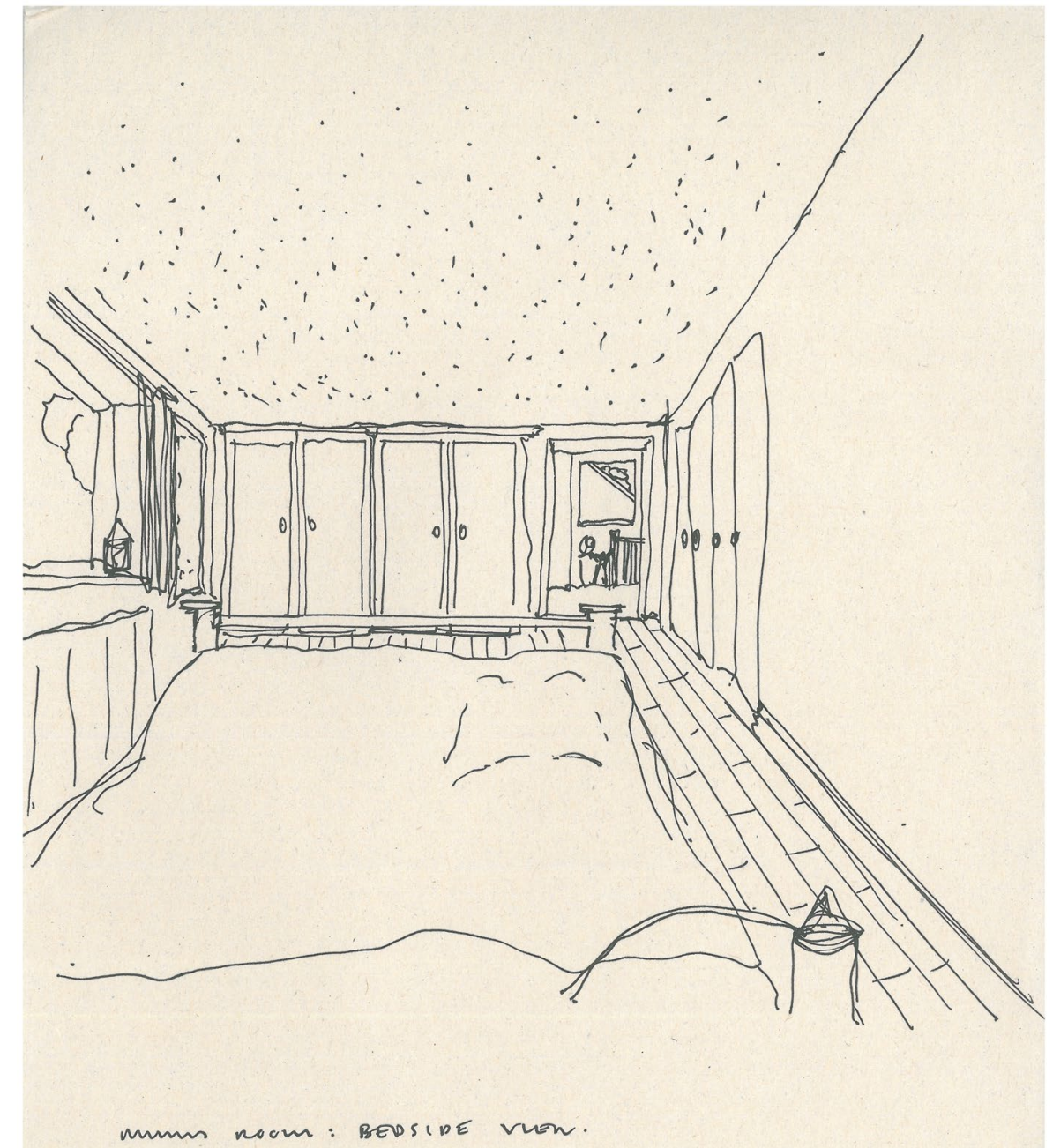




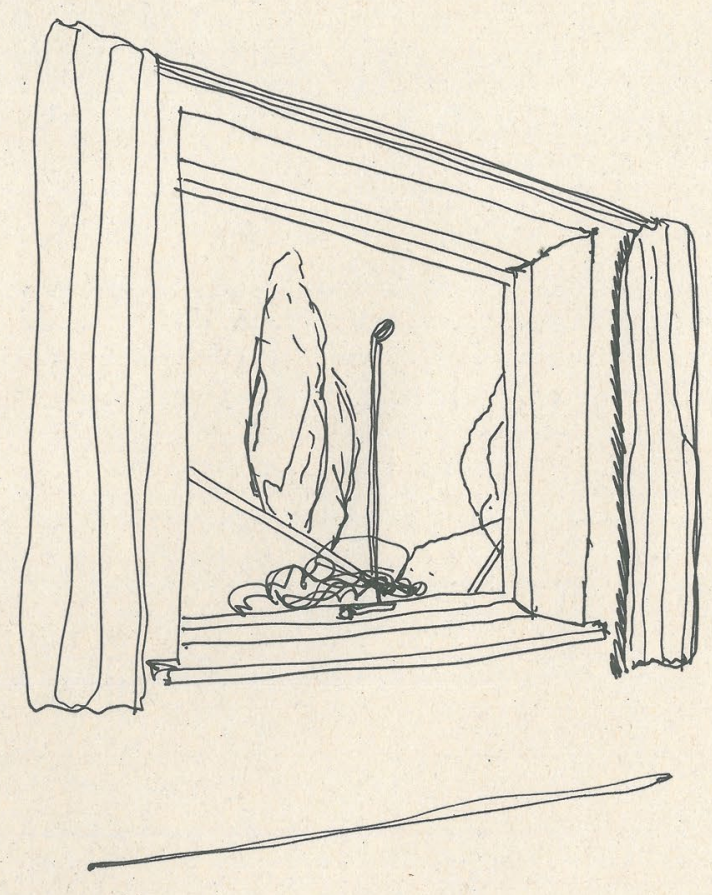




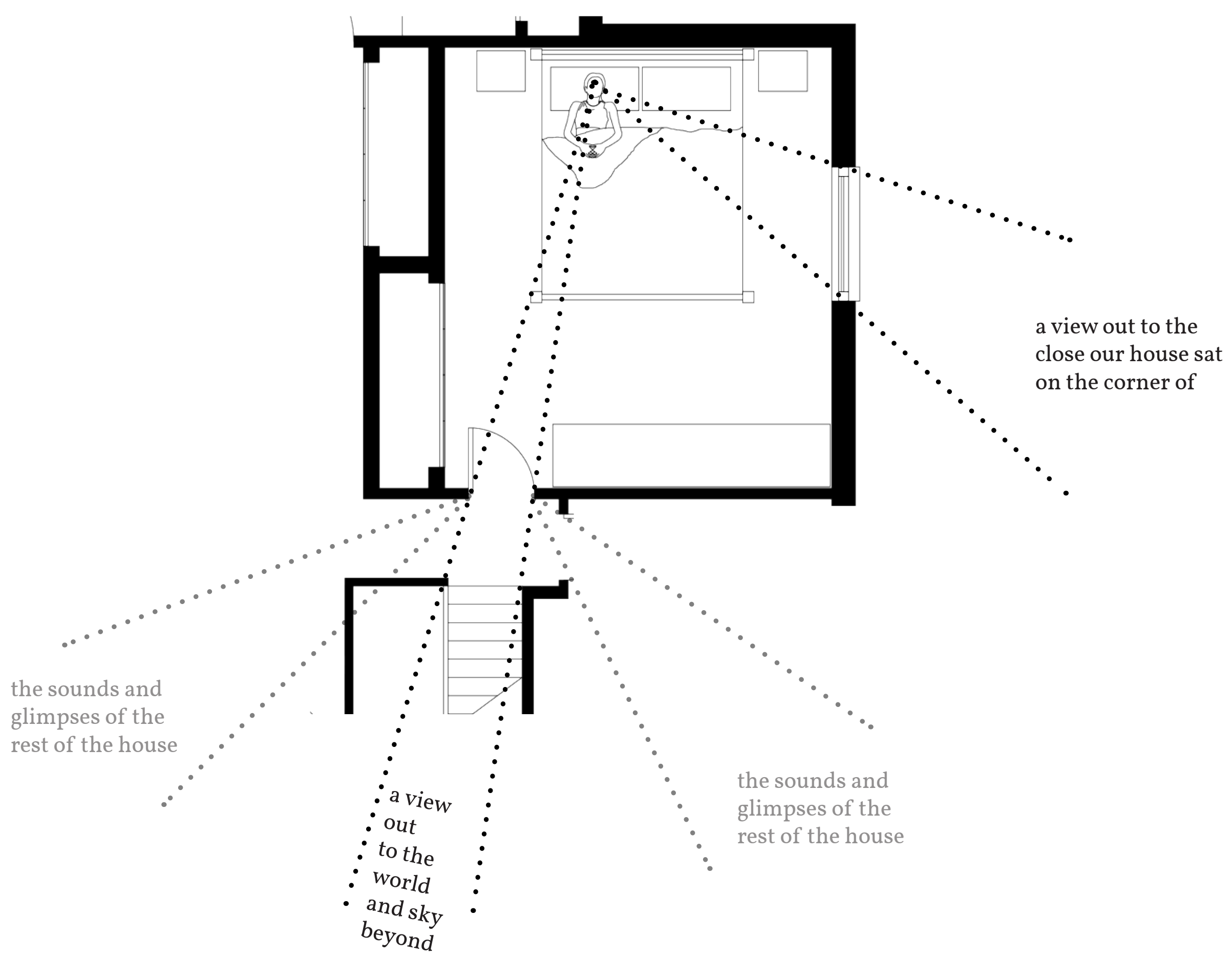




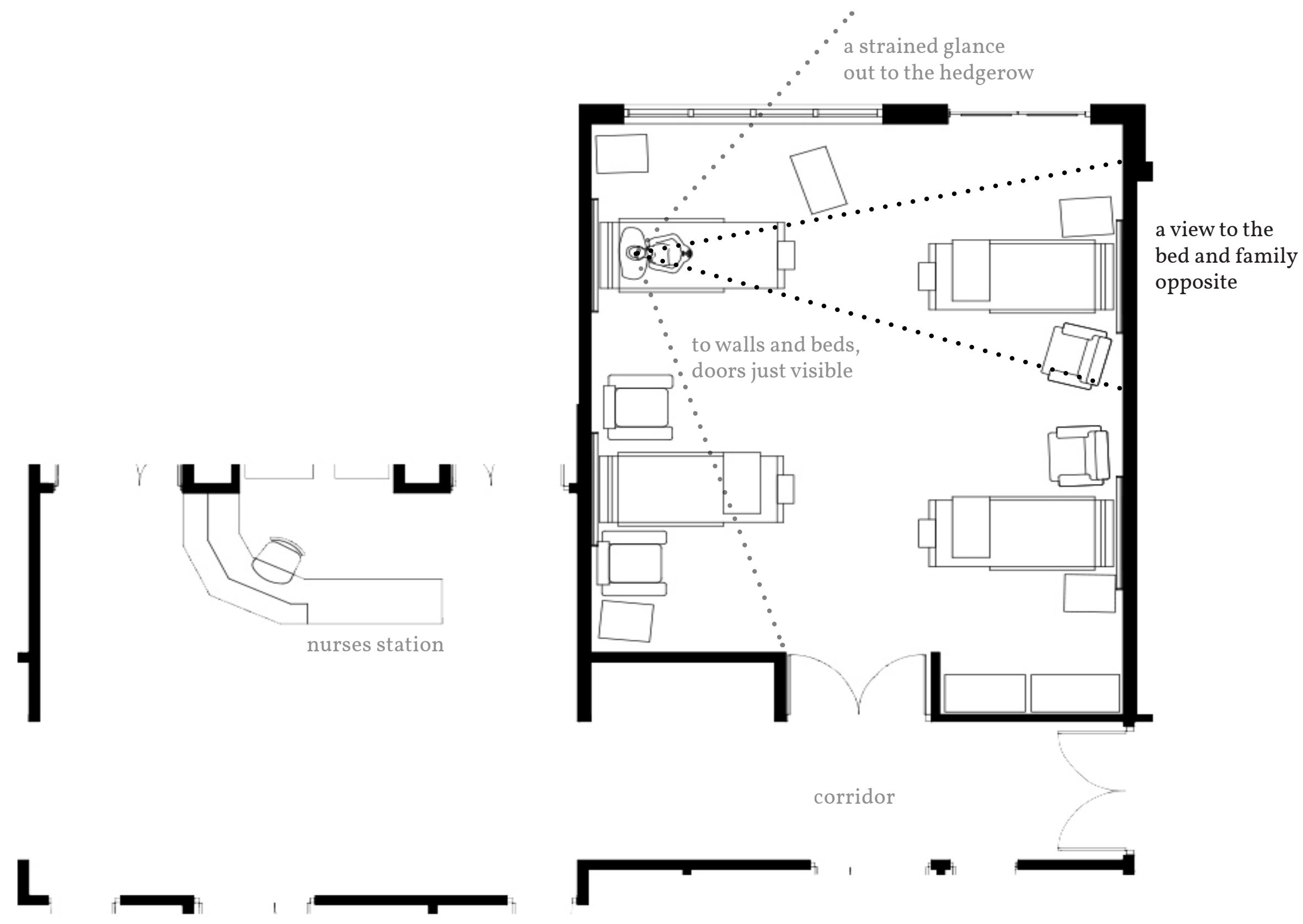



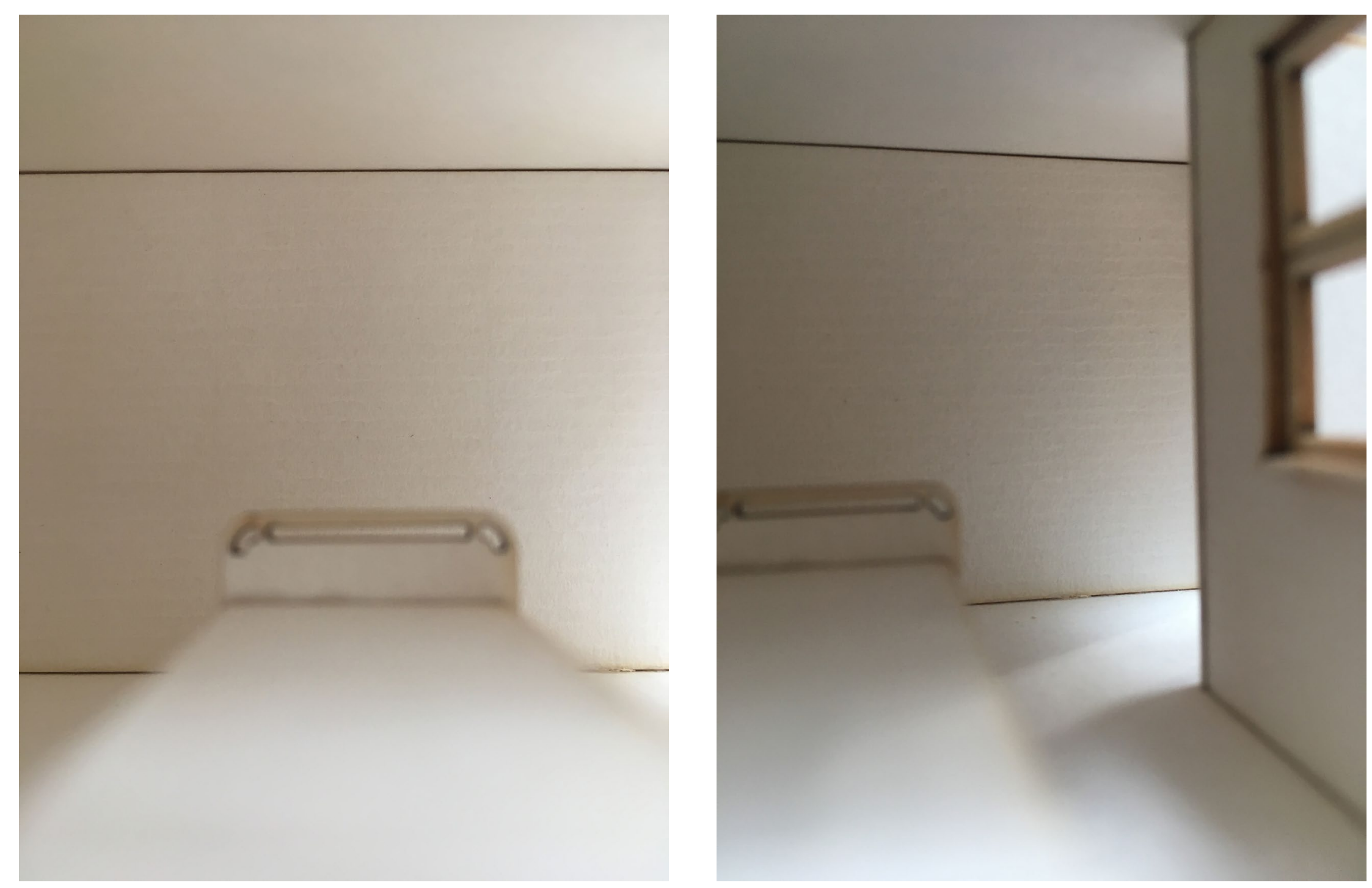

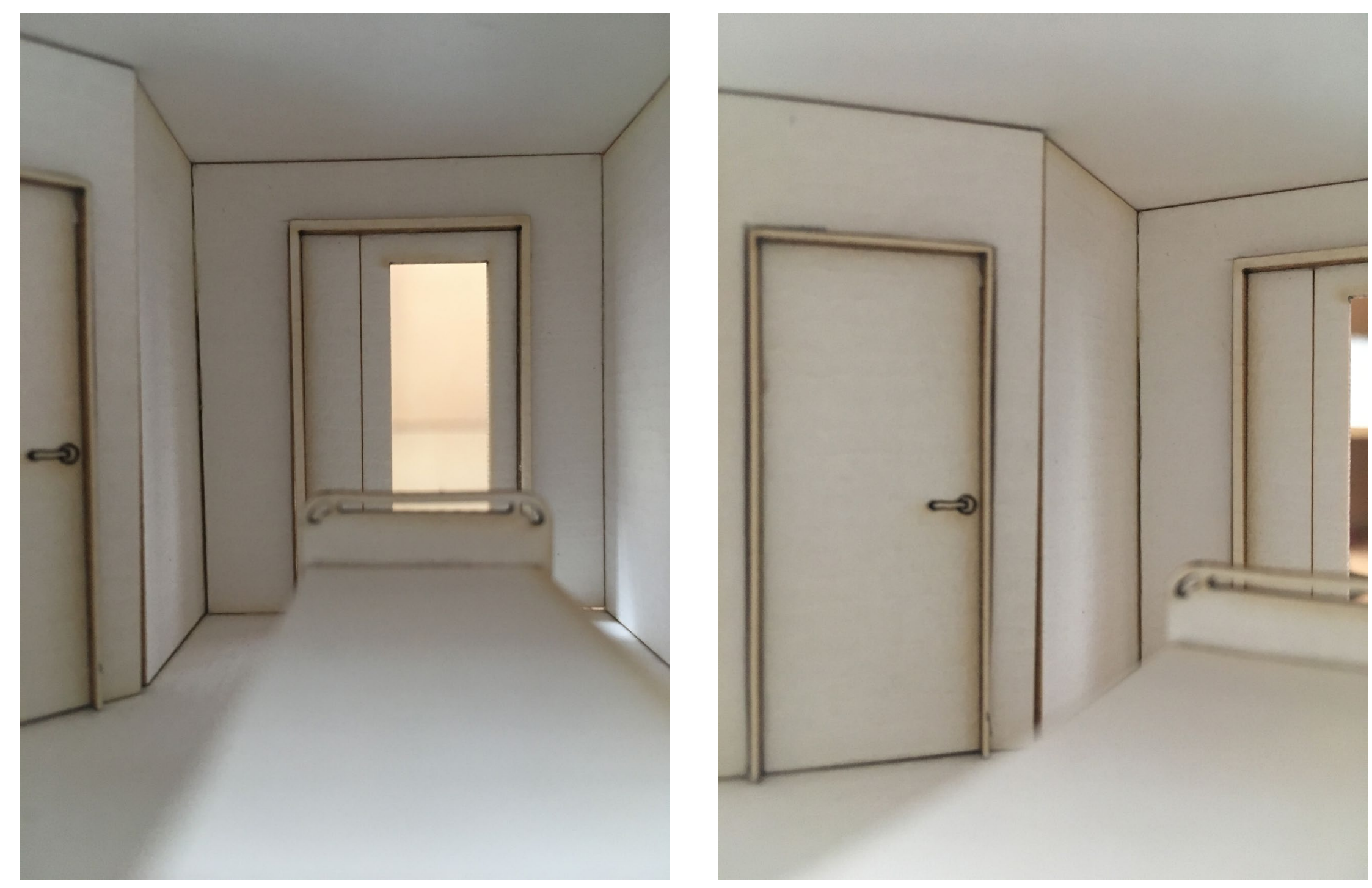


\section{I am what is around me}

Wallace Stevens, Collected Poems 